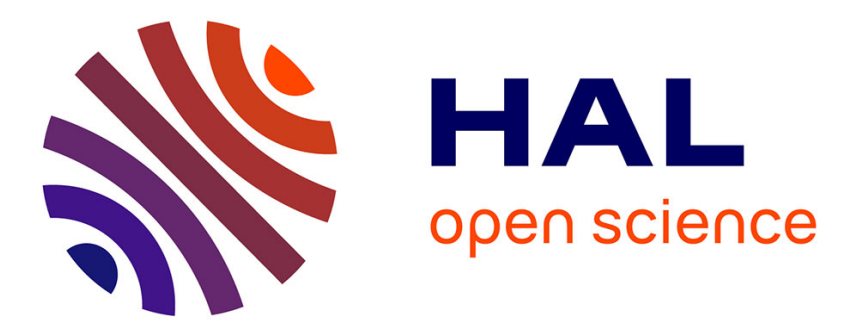

\title{
Analyse des comportements spectraux dans l'olivette de Sfax (Tunisie)
}

Charles Baldy, Frédéric Baret, Ahmed Trigui

\section{To cite this version:}

Charles Baldy, Frédéric Baret, Ahmed Trigui. Analyse des comportements spectraux dans l'olivette de Sfax (Tunisie). Agronomie, 1986, 6 (10), pp.941-948. hal-00884843

\section{HAL Id: hal-00884843 \\ https://hal.science/hal-00884843}

Submitted on 1 Jan 1986

HAL is a multi-disciplinary open access archive for the deposit and dissemination of scientific research documents, whether they are published or not. The documents may come from teaching and research institutions in France or abroad, or from public or private research centers.
L'archive ouverte pluridisciplinaire HAL, est destinée au dépôt et à la diffusion de documents scientifiques de niveau recherche, publiés ou non, émanant des établissements d'enseignement et de recherche français ou étrangers, des laboratoires publics ou privés. 


\title{
Analyse des comportements spectraux dans l'olivette de Sfax (Tunisie)
}

\author{
Charles BALDY, Frédéric BARET \& Ahmed TRIGUI* \\ I.N.R.A., Station de Bioclimatologie, Centre de Recherches d'Avignon, B.P. YI, F 84140 Montjavet \\ * Institut de l'Olivier TN 3029, Sfax, Tunisie
}

Mots clés additionnels : Signatures spectrales, culture intercalaire.

\section{INTRODUCTION}

L'olivette sfaxienne constitue un ensemble agricole original, développé en climat méditerranéen maritime aride. (BALDY, 1965). (Carte des pluies dans la région de Sfax). Les pluies, inférieures en moyenne à $200 \mathrm{~mm}$ par an, tombent surtout de l'automne au printemps, en peu de jours (15 à 30); souvent orageuses, elles proviennent surtout du secteur est, et plus rarement du nord et du nord-ouest.
L'influence maritime se traduit par des brumes, brouillards et rosées nocturnes et matinaux fréquents (100 à 200 jours par an, selon les années). Ils apportent une quantité d'eau difficilement mesurable, mais dont le rôle pour l'alimentation en eau des arbres est très important.

La région subit une forte influence saharienne quand elle est soumise à des masses d'air sec venant du sud-ouest, qui s'accompagnent souvent de sirocco. Les températures, douces en hiver (la gelée est excep- 


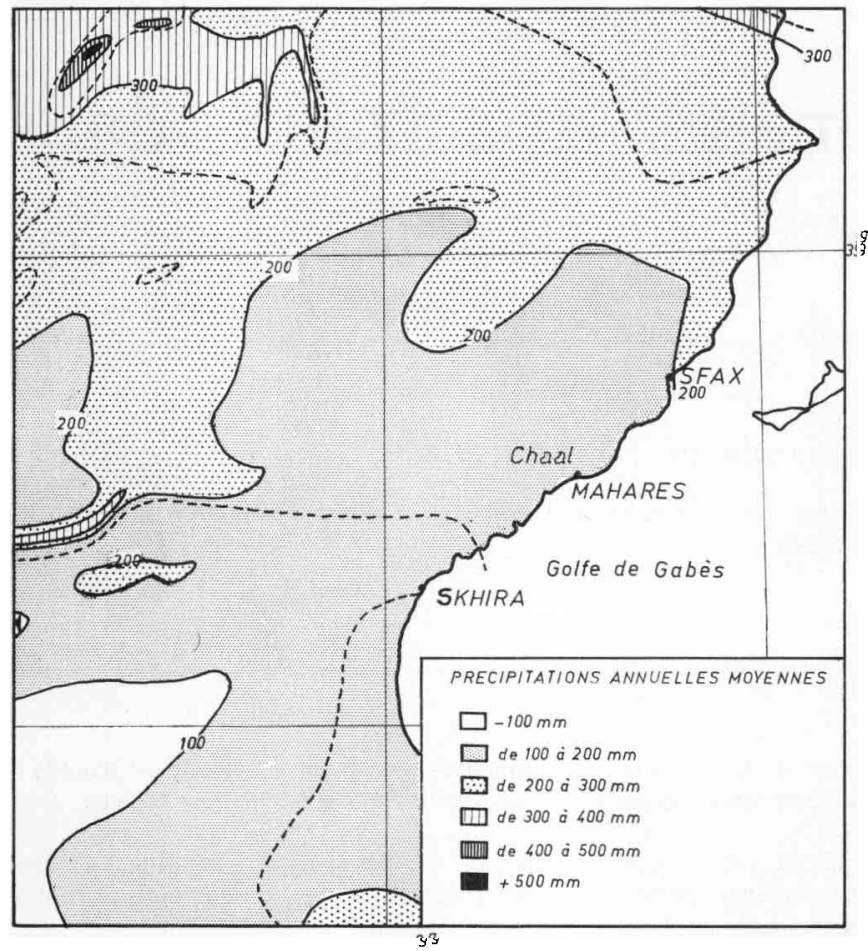

Carte.

Pluies annuelles dans la région de Sfax.

Annual rainfall in the region of Sfax.

tionnelle), peuvent être très élevées en èté, quand la région est sous l'influence des masses d'air saharien.

Les sols, de couleur claire, sont généralement sableux à sablo-argileux, ou sablo-limoneux, parfois gypseux ou salés ; leur $\mathrm{pH}$ est généralement basique à neutre. Leur profondeur est très variable; on a souvent des placages sableux éoliens en surface pouvant donner des endunements : micro-nebka, ou barkhanes. A d'autres endroits, les érosions (éolienne ou hydrique) peuvent dégrader fortement les sols. Selon les cas, les racines pourront être déchaussées (parfois sur plus de $50 \mathrm{~cm}$ ), ou les troncs seront ensablés, sur des profondeurs au moins analogues.

Les sols étaient autrefois découpés par un réseau de tabia, levées de terre faisant clôture, qui rythmaient le paysage, fixaient efficacement les sables errants, et limitaient le ruissellement des pluies d'orage. Elles ont disparu pour la plupart, parfois remplacées par des travaux de défense et restauration des sols (DRS) (fig. 1).

Les vergers ont été plantés pour la plupart entre 1880 et 1920 , le plus souvent à $24 \times 24 \mathrm{~m}$; beaucoup ont actuellement besoin d'être rajeunis, régénérés, voire remplacés. Ce remplacement est souvent réalisé actuellement avec d'autres espèces fruitières (pistachiers, amandiers, plus rarement abricotiers, pêchers, pommiers, parfois vigne de table). Assez souvent, des plantations intercalaires sont réalisées, soit entre des oliviers très jeunes, soit pour rajeunir de vieux vergers. Le taux de recouvrement des sols par les arbres fruitiers dépasse rarement 10 p. 100 dans les conditions de culture de la région.

Malgré l'épuisement des sols, beaucoup de replantations sont effectuées à des distances plus faibles, avec des amandiers et des pistachiers, surtout. On trouve assez souvent aussi des cultures annuelles en bandes

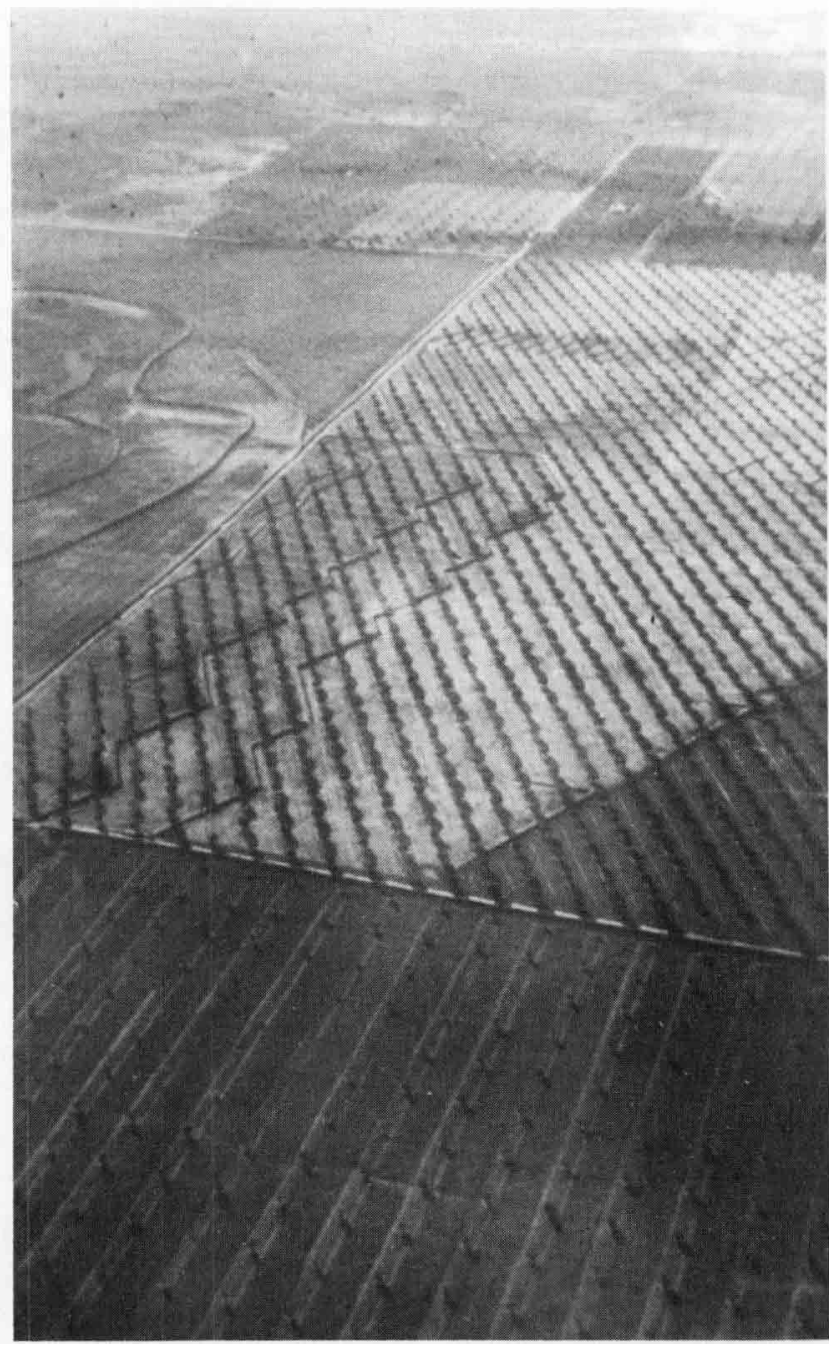

Figure 1

Vue aérienne de la région de Sfax. On remarquera les systèmes de défense et restauration des sols, et la densité de plantation des arbres $(24 \times 24 \mathrm{~m})$.

Aerial view of the region of Sfax. Note the soil protection and defence systems (DRS), the planting density of the orchards $(24 \times 24 \mathrm{~m})$.

dans les interlignes (orge surtout) : cette pratique est courante les premières années de plantation : les éteules sont souvent laissées tout l'été et protègent un peu le sol. D'autres cultures (pastèques, fèves...) se rencontrent parfois. Elles occupent de 20 à 80 p. 100 des sols, pendant le printemps surtout.

Le plus souvent, les sols sont travaillés à plusieurs reprises de façon très superficielle, au cours de l'année; ils sont parfois fortement envahis par le chiendent. Certaines parcelles, plus ou moins abandonnées, sont envahies par une végétation de type steppique : il s'agit surtout de sols gypseux ou salés, aux rendements faibles ou nuls.

Le Domaine du Chaal a été choisi comme site essentiel de l'étude. Couvrant plusieurs milliers d'hectares, il est représentatif de la plupart des milieux et des sols de la région. Les rendements de chaque parcelle y sont enregistrés depuis l'origine des arbres, ce qui permet de mieux comprendre leur comportement.

Une recherche bibliographique ne nous a pas permis de trouver d'exemples d'études menées sur oliviers dans une situation similaire : en Tunisie, des observa- 
tions non publiées avaient montré il y a quelques années que les scènes fournies par les satellites Landsat ne permettaient pas de distinguer les parcelles complantées en oliviers des sols nus.

Nous présentons ici une étude exploratoire, destinée à mettre en évidence le comportement spectral des différentes composantes de l'olivette, c'est-à-dire les oliviers, les autres arbres, les sols, et les cultures annuelles intercalaires.

Une analyse typologique des parcelles aux différentes saisons, pendant quelques années, devrait permettre de mettre en évidence l'évolution des sols due aux pratiques culturales. Les services agronomiques tunisiens ont bien montré déjà qu'elles se traduisent fortement dans la production des vergers.

Pour préparer cette étude, trois campagnes de mesures ont été entreprises, du 25 au 27 septembre 1984, du 27 au 30 mars 1985, et du 20 au 29 mars 1986 : cette dernière n'est pas utilisée ici ; elle a exactement confirmé les données obtenues en mars 1985. Les mesures ont donc été faites pour des positions relatives du soleil identiques. Elles ont pu être réalisées grâce à l'appui financier du Projet régionai oléicole PNUD/FAO, et du Conseil oléicole international (COI).

Son but final sera d'examiner comment assurer un suivi régulier de l'ensemble de la région par télédétection satellitaire, et en particulier combien d'images à haute résolution seront nécessaires chaque année pour obtenir des résultats bien représentatifs. Le suivi systématique de zones-témoins par imagerie satellitaire à haute définition ne se justifiera que si elle présente de moindres frais que l'observation au sol. Nous chercherons à tester ce point dans une deuxième phase du travail.

\section{MATÉRIEL ET MÉTHODES}

\section{A. Mesures radiométriques}

L'étude de terrain a été réalisée à l'aide de radiomètres de marque CIMEL Electronique, de mêmes caractéristiques que ceux qui sont embarqués sur le satellite SPOT 1. Ce matériel a été décrit par GUYOT et al. (1983). Il comporte deux têtes de mesure indépendantes et identiques, permettant la mesure simultanée de la luminance et de l'éclairement. La figure 2 présente le matériel.

Les bandes spectrales suivantes sont utilisées : elles correspondent à celles de SPOT, et sont approximativement comparables à celles de Landsat :

\begin{tabular}{|c|c|c|}
\hline $\begin{array}{l}\text { Bandes } \\
\text { de SPOT }\end{array}$ & Longueurs d'onde & $\begin{array}{l}\text { Bandes } \\
\text { Landsat }\end{array}$ \\
\hline - & - & - \\
\hline $\mathrm{S}_{1}$ & 510 à $580 \mathrm{~nm}$ (vert-jaune) & MSS \\
\hline $\mathrm{S}_{2}$ & 630 à $690 \mathrm{~nm}$ (orangé-rouge) & MSS \\
\hline $\mathrm{S}_{3}$ & $\begin{array}{l}790 \text { à } 890 \mathrm{~nm} \text { (proche infra- } \\
\text { rouge) }\end{array}$ & MSS \\
\hline
\end{tabular}

L'ensemble est fixé sur un mât à potence démontable léger, de $2,60 \mathrm{~m}$ de haut, qui permet un champ de vision de $1 \mathrm{~m}^{2}$ pour l'angle d'ouverture de $12^{\circ}$ de la tête de mesure de la luminance. Un cardan permet aux têtes de rester verticales. Les radiomètres sont étalon-

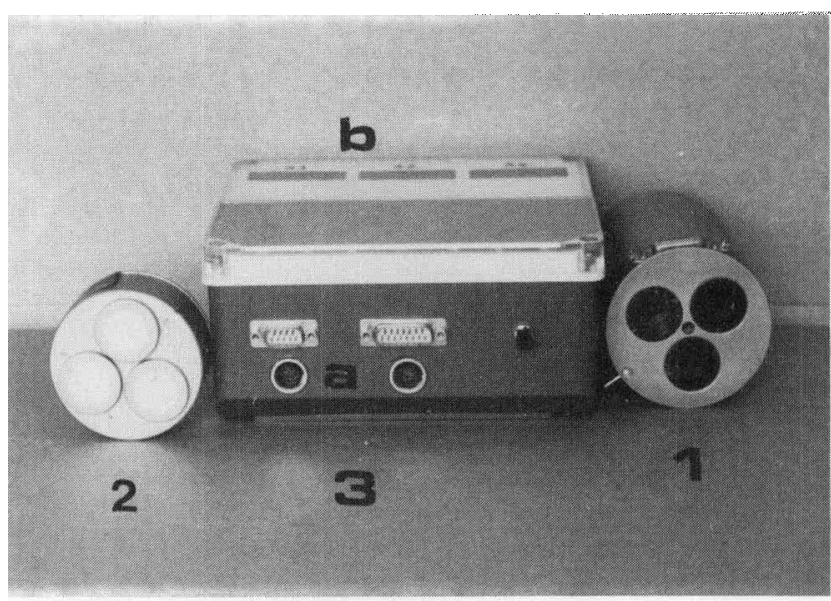

Figure 2

Le radiomètre de simulation de SPOT utilisé : (1) : tête de mesure de luminance; (2) : tête de mesure d'éclairement : on remarque les trois capteurs spectraux ; (3) : boittier de mesure et d'alimentation. On notera les connections vers les capteurs (a), et les fenêtres d'affichage (b).

Radiometer: I) luminance meter ; 2) lightness meter (note the three sensors in both cases); 3) measurement and electric supply box. Note a) sensor connections, b) digitalized counters.

nés à intervalles de temps réguliers, selon la méthode mise au point par GUYOT et VERBRUGGHE (1982).

Pour mesurer les réflectances des oliviers, on a hissé le mât sur une échelle de récolte. Selon les cas, le radiomètre se situera entre $0,50 \mathrm{~m}$ et $1,50 \mathrm{~m}$ du sommet des arbres. La figure 3 présente l'appareillage au champ. La tête de luminance verra, selon les cas, surtout le sol, l'ombre des arbres, ou la végétation, et la tête d'éclairement est placée au soleil, en position aussi dégagée que possible : il est évident cependant que pour les mesures d'ombre, le montage actuel ne permet pas de mesurer le rayonnement incident de façon aussi dégagée qu'il serait souhaitable.

En chaque point, on a effectué 10 à 15 mesures successives, en tournant la potence d'est en ouest, par le sud, pour intégrer la variabilité liée à la position relative du soleil et des ombres portées au sol, ou dans la végétation. Toutes les mesures ont été faites entre 10 et $15 \mathrm{~h}$ TU. La figure 4 schématise la méthode de mesure sur les différents cas rencontrés (sol nu ou cultivé, oliviers ou autres arbres...).

\section{B. Eléments du paysage étudiés}

Les cas étudiés représentent les principales situations rencontrées sur le terrain en 1984 et 1985: tableau 1.

Pour chaque cas, on a étudié des sols de textures différentes, sans chercher à établir une typologie pédologique, des oliviers (taillés récemment ou non), de diverses vigueurs ou subissant des techniques culturales différentes, des céréales fournies ou clairsemées, vertes ou sèches... Les «autres arbres » regroupent pistachiers, amandiers, vignes et cactus. Sauf pour les oliviers à fort développement, la contribution de la réflectance du sol (plus ou moins ombragé) joue un rôle important, car les indices foliaires sont généralement faibles. 


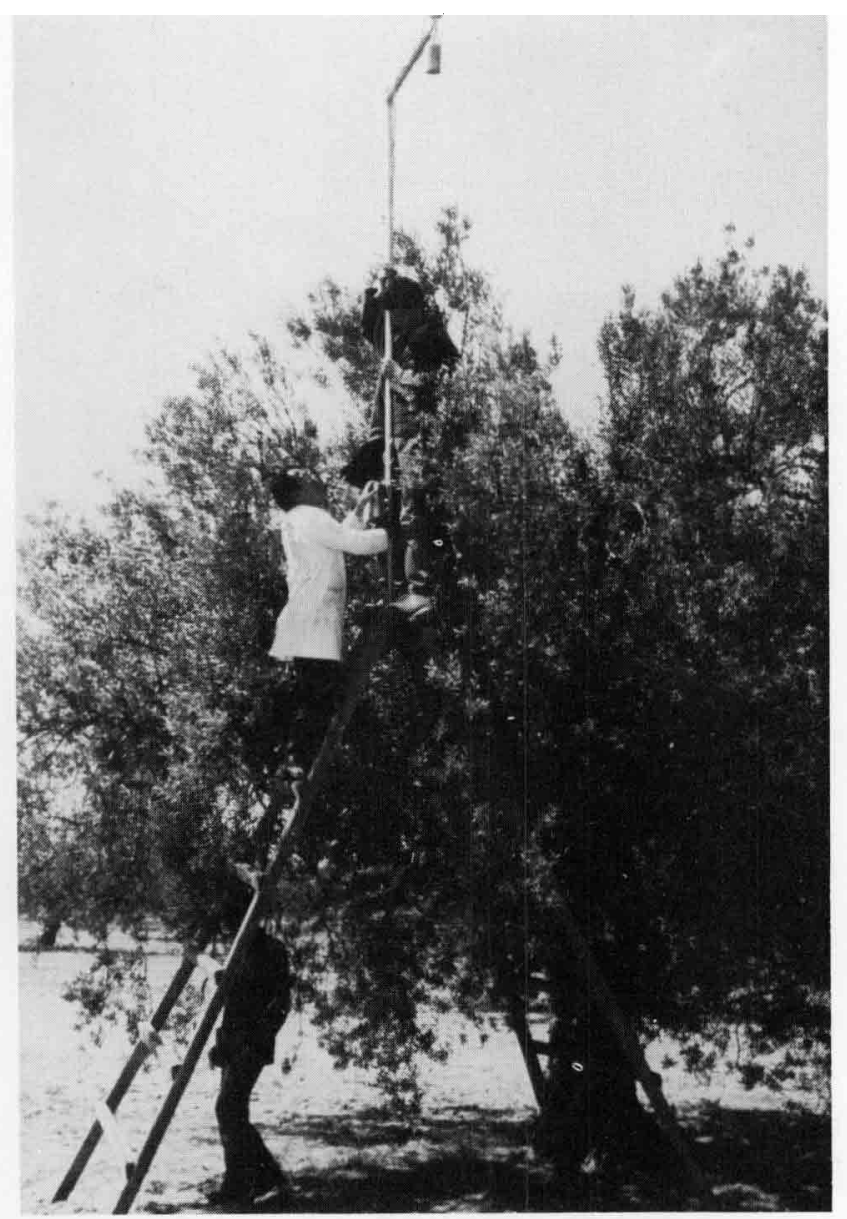

Figure 3

Mesure des réflectances sur olivier. On notera le mât-support, les têtes de mesure, et la bonne verticalité de l'ensemble.

Reflectivity measurements over an olive tree. Note the mast, the measuring heads and the good vertical alignment.

TABLEAU 1

Nombre de cas étudiés. Chacun a comporté 5 à 15 mesures par site. Number of cases studied (5-15 measurements per site).

\begin{tabular}{lll}
\hline \multicolumn{1}{c}{ Années } & 1984 & 1985 \\
\hline 1. Sols nus travaillés & 8 & 7 \\
2. Sols nus non travaillés & 6 & 6 \\
3. Sols nus à l'ombre & 7 & 6 \\
4. Végétation de la Sebkha & 1 & 2 \\
5. Réflectance des oliviers & 3 & 14 \\
6. Steppe, chemins de terre... & 2 & 4 \\
7. Céréales (orge...) & 1 (éteules) & 3 (vert) \\
8. Chiendent & 3 & 0 \\
9. Autres arbres, cactus, vigne... & 4 & 1 \\
& & \\
\hline
\end{tabular}

\section{RÉSULTATS EXPÉRIMENTAUX}

\section{A. Analyses des mesures correspondant aux sols nus}

On a regroupé dans le tableau 2 les sols nus, travaillés récemment (cas 1 du tableau 1) ou non (cas 2), et (cas 3), l'ombre portée par des troncs, des murs, des cactus ou les cimes d'oliviers très feuillues. On ne reporte ici que les relations entre les bandes :

Les corrélations entre les bandes S1, S2 et S3 du tableau 2 sont très étroites, en particulier entre $\mathrm{S} 1$ et

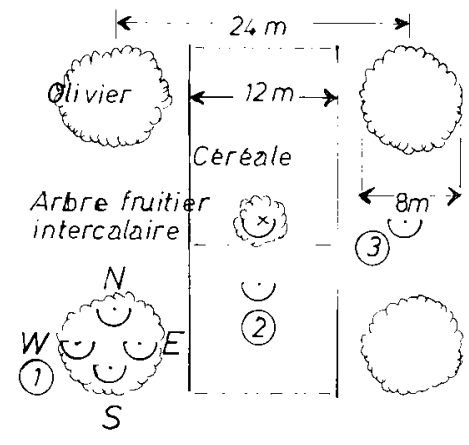

Figure 4

Méthode de mesure des réflectances sur les différents milieux dans l'olivette de Sfax. On a figuré par des demi-cercles les séries de dix à quinze mesures, faites dans chaque cas d'est en ouest : (1) : sur les oliviers ; (2) : sur céréale ; (3) sur sol nu entre les arbres.

Method for measuring the reflectivity of the different types of ground cover in the olive orchards. Half circles show the ten to fifteen measures made in each series, always from east to west 1) over olive tree ; 2) over cereal ; 3) over bare soil, between trees.

TABLEAU 2

Matrice des corrélations du sol nu, travaillé récemment ou non, et de l'ombre.

Correlation matrix of bare soil (recently ploughed and Shade).

\begin{tabular}{cccc} 
Variables & S1 & S2 & S3 \\
\hline S3 & 0,928 & 0,932 & 1,000 \\
S2 & 0,988 & 1,000 & \\
S1 & 1,000 & & \\
\hline
\end{tabular}

S2 $(r=0,988)$. Il n'y a par contre aucune relation entre la différence (S3 - S2) et les bandes visibles $\mathrm{S} 1$ et $\mathrm{S} 2$.

Les rapports $\mathrm{S} 2 / \mathrm{S} 1$, et $\mathrm{S} 3 / \mathrm{S} 2$ sont peu corrélés avec les variables simples : en particulier, $\mathrm{S} 3$ et la différence normalisée $\mathrm{DN}=(\mathrm{S} 3-\mathrm{S} 2) /(\mathrm{S} 3+\mathrm{S} 2)$, ou le rapport $\mathrm{R}=\mathrm{S} 3 / \mathrm{S} 2(-0,177)$.

La brillance $\mathrm{BR}$ est très fortement corrélée avec les trois bandes spectrales, et avec la différence (S2 - S1): Il s'agit de la relation proposée par SAINT, PODAIRE, ANGLADE (1981), définie comme l'indice de brillance des sols nus : $B R=\sqrt{(\mathrm{S} 3)^{2}+(\mathrm{S} 2)^{2}}$.

Le rapport $\mathrm{R}=\mathrm{S} 3 / \mathrm{S} 2$ ou droite des sols aboutit à la régression suivante : $\mathrm{S} 3=1,24 \mathrm{~S} 2 \pm 7$ p. 100 , avec une corrélation de 0,932 .

La figure 5 présente l'effet dû à quelques types de sols très différenciés (comparer les sols 3 et 8 ) : on a mis en évidence aussi l'effet de la position relative du soleil et des ondulations dues au travail du sol (VANDERBILT et al., 1981 ; GUYOT, 1983).

La figure 6 présente l'ensemble des rapports S3/S2 pour les sols nus et l'ombre des arbres. Il s'agit des moyennes et des écarts-types $\sigma$ pour l'ensemble des mesures.

\section{B. Analyse des mesures correspondant à la végétation}

\section{Comparaison de divers indices}

Parmi les nombreuses combinaisons possibles des canaux, on a choisi les suivantes, toutes décrites dans 


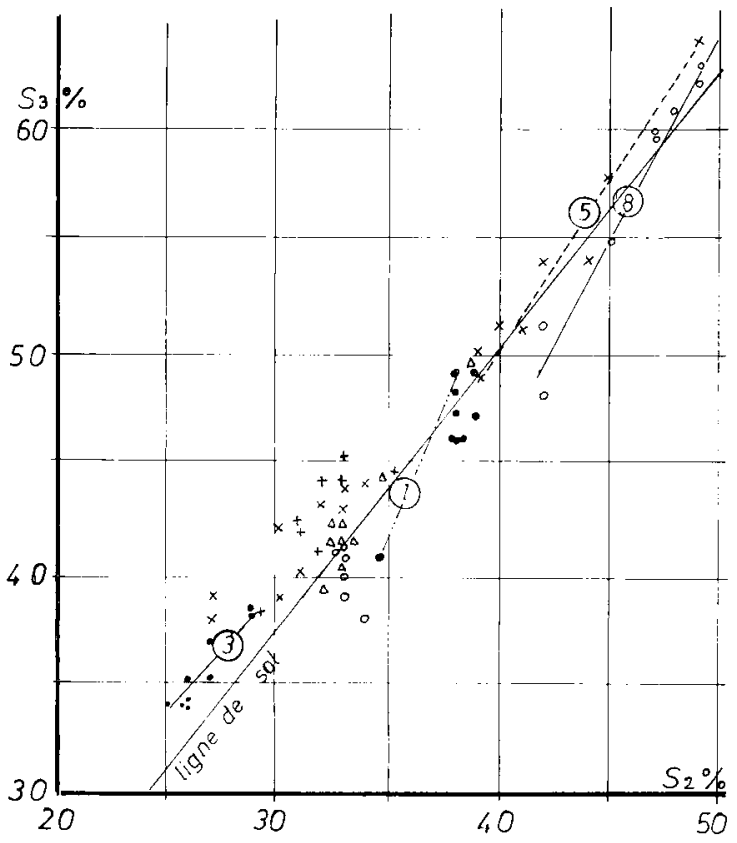

Figure 5

Variabilité des réflectances rouge et proche infra-rouge sur les sols, travaillés récernment ou non : (3), (5), (8) correspondent à trois sites étudiés, bien différenciés en valeurs absolues des réflectances.

Variability of red and infra-red reflectivity over bare soils, recently ploughed or not. Soils 3, 5 and 8 are in three areas with clearly distinct characteristics.

la monographie de BARIOU et al. (1985), et on a calculé les matrices des corrélations (tabl. 3).

Les bandes $\mathrm{S} 1$ et $\mathrm{S} 2$ sont très étroitement corrélées ; par contre $\mathrm{S} 1$ (ou S2) et $\mathrm{S} 3$ le sont très peu, sauf dans le cas du sol. On n'a donc retenu que les combinaisons de S2 et S3 dans l'exposé ci-dessous.

On a calculé les rapports, les sommes et les différences des trois bandes, prises deux par deux ; en particulier le rapport $\mathrm{S} 3 / \mathrm{S} 2$, et la différence normalisée DN (ou indice de végétation normalisé IVN) : ces deux indices s'avérant très étroitement corrélés dans le cas présent, on n'a utilisé ici que DN.

On a calculé ainsi les indices «perpendiculaires » de RICHARDSON \& WIEGAND (1977) (PVI), destinés à comparer le sol et la végétation à son voisinage et l'indice de brillance «sol » de WIEGAND (BVI). Ces méthodes ne nous ont pas donné de résultats significativement meilleurs que l'indice de végétation norma-

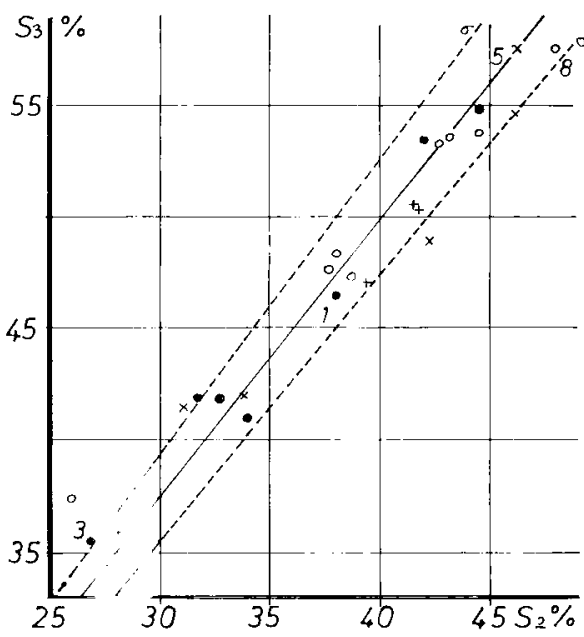

Figure 6

Etude de la régression linéaire de $\mathrm{S3}$ en $\mathrm{S} 2$ pour les sols et l'ombre: moyenne des séries de 10 mesures faites en $1984(0),(+)$, et 1985 $(\bullet),(x)$ sur sol travaillé $(O),(\bullet)$ ou non $(+),(x)$.

Linear regression of $S 3$ on $S 2$ for soils and shadow : mean of series of ten measurements made in $1984(0),(+)$, and $1985 ;(\bullet),(\times)$ over soils : (O), (๑) ploughed, or $(+),(\times)$ not.

lisé, ou le rapport $\mathrm{S} 3 / \mathrm{S} 2$, bien que DE WISPELAERE \& PeYre de Fabregues (1985) en aient montré l'intérêt sur des sols quasi désertiques. Nous ne les utilisons donc pas ici. Soulignons cependant que si les indices retenus sont les plus intéressants dans le cas de mesures au sol, cela ne signifie nullement que d'autres relations ne s'avéreront pas utiles lorsqu'on passera de la simulation à l'étude de scènes fournies par le satellite.

Le tableau 3 présente la matrice des corrélations pour l'ensemble des 680 valeurs analysées.

2. Possibilités de discrimination des différents éléments du paysage et de leur état, à partir de données radiométriques

L'analyse globale est présentée à la figure 7 . On retrouve la droite des sols. On a distingué en 3a l'ombre des oliviers, et en $3 \mathrm{~b}$ la régression pour toutes les ombres ensemble : elles tendent vers les droites des cas comportant de la végétation.

Les droites 4,6 et 8 correspondent à des recouvrements faibles des sols par la végétation naturelle (ou le

TABLEAU 3

Matrice des corrélations.

Correlation matrix.

\begin{tabular}{|c|c|c|c|c|c|c|c|c|c|c|c|}
\hline Valeurs & 1 & 2 & 3 & 4 & 5 & 6 & 7 & 8 & 9 & 10 & 11 \\
\hline $1, \mathrm{SI}$ & 1,000 & & & & & & & & & & \\
\hline $2, \mathrm{~s} 2$ & 0,987 & 1,000 & & & & & & & & & \\
\hline $3, \mathrm{~S} 3$ & 0,704 & 0,658 & 1,000 & & & & & & & & \\
\hline $4, \mathrm{~S} 2-\mathrm{S} 1$ & 0,927 & 0,975 & 0,566 & 1,000 & & & & & & & \\
\hline 5, S3-S2 &,- 418 & $-\quad, 489$ & 0,336 &,- 564 & 1,000 & & & & & & \\
\hline $6, \mathrm{~S} 2 / \mathrm{S} 1$ & 0,601 & 0,703 & 0,189 & 0,813 &,- 660 & 1,000 & & & & & \\
\hline $7, \mathrm{~S} 3 / \mathrm{S} 2$ &,- 606 &,- 643 &,- 021 &,- 666 & 0,779 &,- 733 & 1,000 & & & & \\
\hline $8, \mathrm{DN}$ &,- 751 &,- 793 &,- 137 &,- 817 & 0,833 & 0,783 & 0,883 & 1,000 & & & \\
\hline $9, \mathrm{BR}$ & 0,866 & 0,837 & 0,962 & 0,762 & 0,068 & 0,383 &,- 023 &,- 382 & 1,000 & & \\
\hline $10, \mathrm{BVI}$ & 0,926 & 0,907 & 0,914 & 0,843 &,- 076 & 0,485 &,- 359 &,- 504 & 0,989 & 1,000 & \\
\hline 11, PVI & 0,560 &,- 625 & 0,163 &,- 688 & 0,971 &,- 726 & 0,809 & 0,861 & 0,104 & 0,246 & 1,000 \\
\hline
\end{tabular}




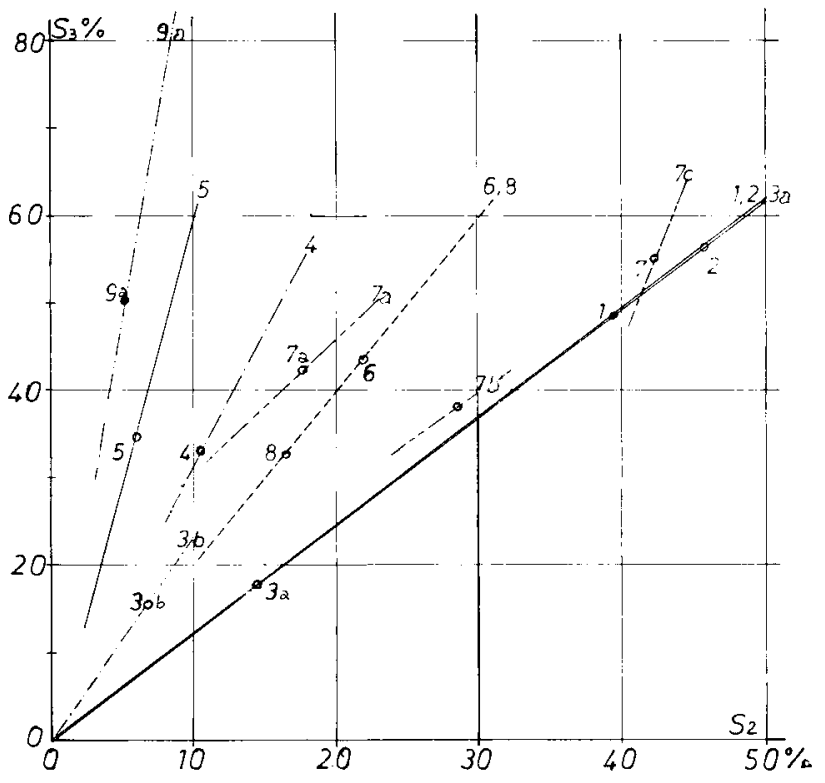

Figure 7

Régressions linéaires de S3 en S2 pour les 9 cas considérés.

Linear regressions of $S 3$ on $S 2$ for the nine cases studied.

chiendent). La végétation de la Sebkha, alimentée en eau salée, est plus dense que celle de la steppe.

On a distingué en 7 l'orge verte et dense (7a), l'orge en éteules sèches (7b), et de l'orge verte, mais très dispersée sur le sol (7c); ces deux dernières se rapprochent de la droite des sols. On a porté en 5, la régression générale par les oliviers, et en 9a celle qu'on a obtenu pour les amandiers.

\section{Le comportement spectral des oliviers}

On a utilisé, d'une part, le rapport $\mathrm{R}=\mathrm{S} 3 / \mathrm{S} 2$, et d'autre part, la différence normalisée DN pour caractériser celui-ci.

Le tableau 4 présente les corrélations entre les paramètres retenus. On peut constater que $\mathrm{R}$ et $\mathrm{DN}$ sont très étroitement liés, mais que la Brillance BR est indépendante de ces deux derniers indices.

TABLEAU 4

Corrélations pour l'olivier.

Correlations for olive.

\begin{tabular}{lrrrrrr}
\hline \hline Variables & S1 & S2 & S3 & R & DN & BR \\
\hline S1 & 1,000 & & & & & \\
S2 & 0,973 & 1,000 & & & & \\
S3 & 0,460 & 0,332 & 1,000 & & & \\
R &,- 594 &,- 665 & 0,177 & 1,000 & & \\
DN &,- 805 &,- 882 & 0,049 & 0,891 & 1,000 & \\
BR & 0,676 & 0,572 & 0,963 &,- 015 &,- 199 & \\
\hline
\end{tabular}

La majorité des points figurés en (3) figure 8 , qui regroupe toutes les mesures individuelles de réflectance des oliviers, s'alignent étroitement sur une relation du type : $\mathrm{S} 2=0,2( \pm 0,1) \mathrm{S} 3$.

En (2), on trouve des mesures faires sur des «trous " dans les houppiers, ou sur des arbres fortement taillés : la contribution du flux réfléchi par le sol est plus

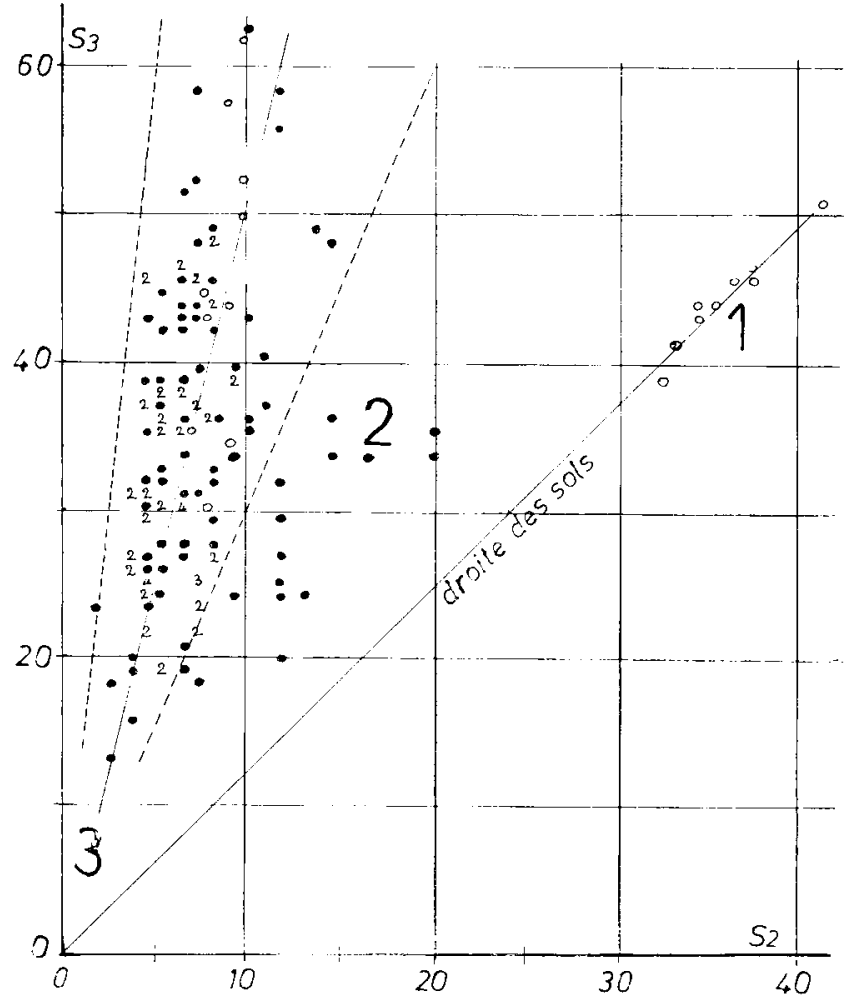

Figure 8

Valeurs individuelles de réflectance des oliviers : on a distingué trois cas : (I): oliviers morts; (2): mesures sur des couverts peu couvrants; (3) : couverts couvrants.

Reflectivity of individual olive tres. Distinguish three cases: (I) dead trees; (2) light cover; (3) dense cover.

ou moins forte. En (1), on a des oliviers sénescents ou morts, mais qui portaient encore leurs feuilles, grisâtres : ils s'alignent sur la droite des sols. Quand plusieurs points se superposent, on a indiqué leur nombre : $2,3,4 \ldots$

On présente sur la figure 9 l'analyse fréquentielle des différences normalisées DN pour l'ensemble des mesures faites sur les oliviers : on distingue bien trois classes : les arbres plus ou moins morts, qui ont des DN de sol nu (1), les mesures sur arbres peu denses (2) (DN de 0,2 à 0,4 ), et les mesures sur feuillage dense (3) (DN de 0,5 à 0,9 ). Les valeurs indiquées (4) signalent des mesures faites sur de jeunes arbres: ils se classent bien comme les arbres "normaux», mais leur faible développement posera un problème de reconnaissance par satellite...

La figure 10 analyse les valeurs individuelles des différences normalisées $\mathrm{DN}$ pour un olivier bien développé, sur lequel nous avons effectué quatre séries de mesures, par les quatre faces, comme schématisé sur la figure 4. L'échelle des abscisses est très étalée, par rapport à la figure 9 . On a indiqué par $(\mathrm{N}),(\mathrm{S}),(\mathrm{E})$, (W), les moyennes des quatre séries de dix mesures, et par (M) la moyenne générale de l'arbre. On notera les deux classes d'indices : $(0,5$ à 0,6$)$, où le sol apparaît, et $(0,6$ à 0,8$)$, caractéristiques d'une végétation abondante.

L'orientation relative des capteurs et du soleil, la position des feuilles (faces supérieure verte, ou inférieure blanchâtre dominantes) (TRIGUI, 1983), expliquent cette variabilité dans les classes d'indices. 


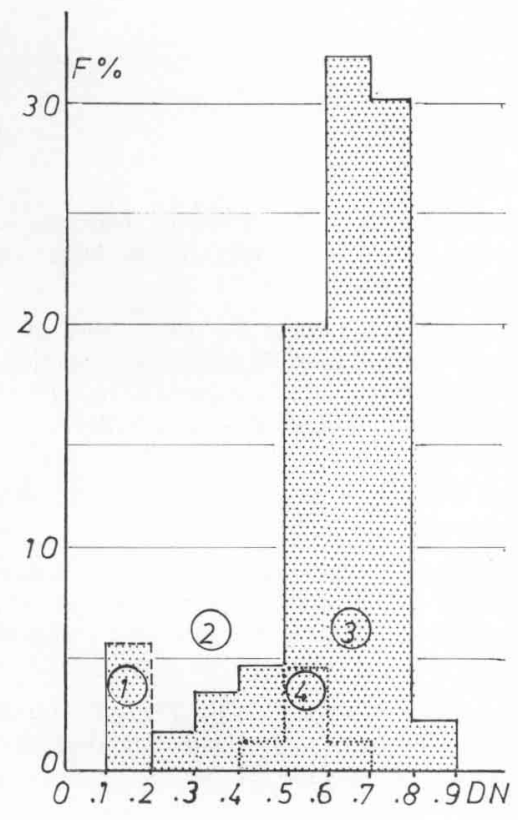

Figure 9

Variabilité des valeurs individuelles mesurées sur les oliviers, exprimée en différences normalisées: on distingue bien les trois classes de recouvrement.

Variability of individual values obtained over the canopies of olive trees, expressed as normalized differences. The three density classes are readily distinguished.

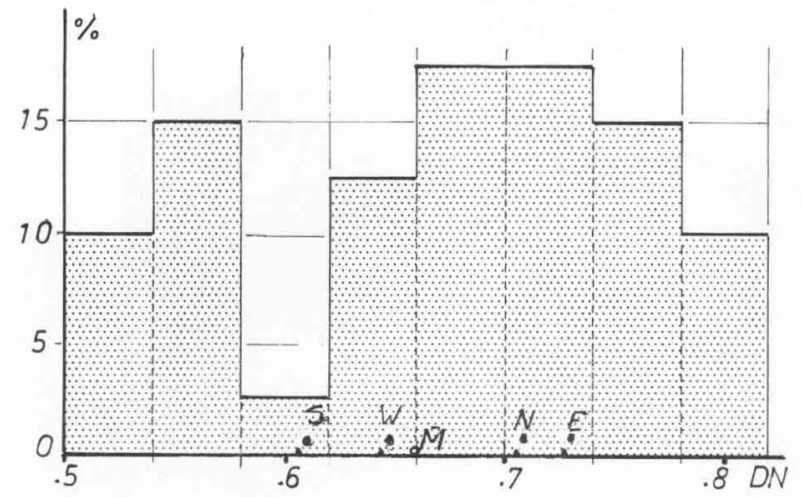

Figure 10

Variabilité des $D N$ pour un olivier : quatre séries de mesures sont faites aux quatre directions $N, S, E, W$ : on observe deux classes de réflectances.

Variability of the DN for an olive tree. Four series of measurements are made, in the $N, S, E, W$, directions, and two reflectivity classes obtained.

Une certaine incertitude existe encore en ce qui concerne la représentativité des mesures ponctuelles faites sur les houppiers, par rapport à des arbres entiers : on a pu noter, sur les figures 9 et 10 que la variabilité observée sur une seule cime est comparable à celle de houppiers différents; on peut cependant distinguer des répartitions différentes, selon que les arbres ont été ou non taillés récemment : les mesures entreprises en 1986 le confirment.

\section{EN CONCLUSION}

L'étude radiométrique de l'olivette du Chaal, de ses sols et des formations végétales qu'on y rencontre, met en évidence les différences existant entre leurs comportements spectraux. Les écarts constatés sont assez importants pour qu'on puisse espérer les analyser à partir de scènes satellitaires à haute définition. Ces études préalables montrent aussi que beaucoup de phénomènes ne pourront être perçus par télédétection spatiale qu'en comparant les mêmes situations à des saisons différentes, et en mettant au point des méthodes d'analyse adaptées aux conditions régionales : tel est le cas de la mise en évidence des oliviers (arbres à feuilles persistantes) par rapport aux autres fruitiers (tous à feuilles caduques), ou de la présence du chiendent (développé en été, mais invisible en hiver).

Les valeurs de réflectance des oliviers sont très différentes, selon qu'ils ont ou non été taillés récemment, et qu'ils vivent dans des conditions plus ou moins défavorables... Les dimensions respectives du pixel de SPOT $(10 \times 10 \mathrm{~m}$ en panchro, et $20 \times 20 \mathrm{~m}$ en multispectral), et des plantations de Sfax $(24 \times 24 \mathrm{~m})$ fait penser que les arbres pris individuellement ne pourront pas être distingués, mais qu'on pourra probablement mettre en évidence leurs alignements, les effets des techniques culturales, de l'érosion ou de l'ensablement des vergers, et de la couverture des sols : il sera probablement nécessaire de compléter l'analyse par des scènes en panchro (pixel de $10 \times 10 \mathrm{~m}$ ), pour préciser certains détails.

Dans le cas des images fournies par SPOT, les ombres portées sur le sol par les arbres à $11 \mathrm{~h}$ du matin représentent, selon la saison, de près de 100 p. 100 de la surface des couronnes en hiver à moins de 50 p. 100 en été. Les arbres occupent, on l'a $\mathrm{vu}$, moins de 10 p. 100 de la surface, le plus souvent. L'orientation des lignes formées par les alignements des arbres et de leur ombre pourront probablement constituer un élément intéressant d'analyse.

Un article ultérieur portera sur l'analyse, à l'aide de scènes SPOT, des effets de l'irrigation et du froid sur des oliviers cultivés à 300 arbres à l'ha environ en Provence et comparera les résultats obtenus dans des conditions climatiques extrêmes de Provence et de Tunisie. 


\section{RÉFÉRENCES BIBLIOGRAPHIQUES}

Baldy Ch., 1965. Climatologie et bioclimatologie de la Tunisie centrale. Projet FAO/FSNU, 200 p. + atlas.

Baldy Ch., 1985. Contributions à l'étude des applications de la Bioclimatologie végétale à l'Agrométéorologie des zones arides et semiarides, en climats méditerranéens et tropical. Thèse Dr. ès-Sciences, Univ. Aix-Marseille, 225 p.

Baret F., Guyot G., 1986. Radiométrie de la maturation de couverts de Blé dans le visible et le proche infra-rouge. Agronomie, 6 (6). 509-516.

Bariou R., Lecamus D., Le Henaff F., 1985. Dossiers de télédétection. Tome 1. Réponse spectrale des végétaux : $71+21 \mathrm{p}$. Tome 2 . Indices de végétation : $121+29 \mathrm{p}$. Tome 3 : Albedo et réflectance: $30+11 \mathrm{p}$. Centre régional de télédétection Editeur. Université de Rennes 2-Haute Bretagne : ISSN 07651120.

Guyot G., 1983. Variabilité angulaire et spatiale des données spectrales dans le visible et le proche infra-rouge. In : $2^{\circ}$ Colloque de signatures spectrales, Bordeaux. Colloques I.N.R.A., $\mathrm{n}^{\circ} 23$, p. 27-44.

Guyot G., Hanocq J. F., Buis J. P., Saint G., 1983. Mise au point d'un radiomètre de simulation de SPOT. In : $2^{e}$ Colloque de signatures spectrales, Bordeaux. Colloques I.N.R.A., n² 23, p. 233-242.
Guyot G., Verbrugghe M., 1982. Compte rendu de l'étalonnage effectué à l'Institut für Lichttechnik, Berlin. Note interne, Bioclimatologie Avignon, $\mathrm{n}^{\circ} \mathrm{M} 82 / 15,23 \mathrm{p}$.

Hanocq J. F., Guyot G., Gruget P., 1981. Mise au point d'un système d'acquisition numérique d'images hémisphériques sous un couvert végétal pour en analyser la structure. In : $l^{\text {er }}$ Colloque de signatures spectrales, Avignon. Colloques I.N.R.A., $n^{\circ}$ 5, p. 391398.

Trigui A., 1983. Propriétés biophysiques et optiques de feuilles d'olivier. Thèse Dr-Ing. USTL Montpellier, 139 p. + figures.

Vanderbilt V. C., Kollenkark J. C., Biehl L. L., Robinson B. F., Bauer M. E., Ranson K. J., 1981. Diurnal changes in reflectance

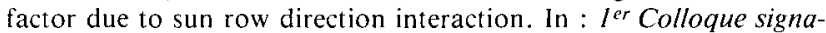
tures spectrales, Avignon. Colloques I.N.R.A., $n^{\circ} 5$, p. 499-508.

Wispelaere G. de, Peyre de Fabregues B., 1985. Action de recherche méthodologique sur l'évaluation des ressources fourragères par télédétection dans la région du Sud-Tamesna (Niger). Note IEMVT/ CIRAD/MRE, $61 \mathrm{p}$. 